\title{
Multimode Network Representation of Two Dimensional Steps in Rectangular Waveguides
}

\author{
A. Alvarez Melcon $\nmid$ and M. Guglielmił
}

†Alcatel Espacio. 28760 Tres Cantos. Madrid. Spain. Fax: 34-1-8040016. Tel: 34-1-8034710. $\ddagger$ European Space Research and Technology Center (ESTEC). P.O. Box 299. Noordwijk, The Netherlands. Fax: 31-1719-84596. Tel:31-1719-83493.

\begin{abstract}
In this paper a new Multimode Network representation for arbitrary rectangular waveguide junctions is presented. The energy coupling between the higher order modes excited in the junction is rigorously described via a multimode impedance matrix representation that can be used to build very efficient and accurate software tools for the analysis of complex structures. The elements of the impedance matrix are obtained from the solution of an integral equation. The main feature of the approach is that the kernel of the integral equation derived is essentially independent from frequency and therefore, the most time consuming task, namely the solution of the integral equation, need to be performed only once per each geometry. The formulation of the method is discussed and the form of the integral equation is given, showing explicitly the dependence with the frequency. A frequency extraction procedure that can lead to a considerable time saving for the analysis of complex structures is also discussed. Finally the design of a commercial communication satellite filter structure is presented, including comparisons between our analysis and measurements of the manufactured hardware.
\end{abstract}

\section{BASIC THEORY}

The Multimode Equivalent Network representation for inductive and capacitive discontinuities in rectangular waveguide has been the subject of previous work [1], [2]. Furthermore, a tiwo dimensional analysis for an arbitrary shaped zero thickness transverse discontinuity in a rectangular waveguide was also presented in [3]. In this paper the theory presented in [1], [2] and [3] is suitably extended to the analysis of arbitrary two dimensional junctions in rectangular waveguides like the one shown in fig. 1. If the fundamental $T E_{1,0}$ mode is incident to this type of discontinuity, the full spectrum of $T M_{m, n}$ and $T E_{m, n}$ modes will be excited. The vector mode functions and the characteristic impedances of the modes are known analytically in both waveguides [4] and therefore, the total transverse magnetic field can be expressed according to the well known modal expansion:

$$
\mathbf{H}_{t}^{(\delta)}=\sum_{m=1}^{\infty} I_{m}^{(\delta) T M} \mathbf{h}_{m}^{(\delta) T M}(x, y)+\sum_{m=1}^{\infty} I_{m}^{(\delta) T E} \mathbf{h}_{m}^{(\delta) T E}(x, y)
$$

where $\delta$ can be 1 for waveguide 1 and 2 for waveguide 2 according to fig. 1 . It is now convenient to observe the behavior of the characteristic impedances at infinity, and define the following admittances.

$$
\begin{gathered}
Y_{m, n}^{(\delta) T M}=\lim _{m, n \rightarrow \infty} Y_{0(m, n)}^{(\delta) T M}=\frac{j \omega \epsilon_{0}}{\sqrt{\left(\frac{m \pi}{a^{(\delta)}}\right)^{2}+\left(\frac{n \pi}{b^{(\delta)}}\right)^{2}}}=j \omega \epsilon_{0} \cdot \hat{Y}_{m, n}^{(\delta) T M} \\
Y_{m, n}^{(\delta) T E}=\lim _{m, n \rightarrow \infty} Y_{0(m, n)}^{(\delta) T E}=\frac{\sqrt{\left(\frac{m \pi}{a^{(\delta)}}\right)^{2}+\left(\frac{n \pi}{b^{(\delta)}}\right)^{2}}}{j \omega \mu_{0}}=\frac{1}{j \omega \mu_{0}} \cdot \hat{Y}_{m, n}^{(\delta) T E}
\end{gathered}
$$

where $Y_{0(m, n)}^{(\delta) T M}$ and $Y_{0(m, n)}^{(\delta) T E}$ are the characteristic admitances for the $T M$ and $T E$ modes respectively.

We can now impose the continuity of the transverse magnetic field in the junction, and write

$$
\sum_{m=1}^{\infty} I_{m}^{(1) T M} \mathbf{h}_{m}^{(1) T M}(x, y)+\sum_{m=1}^{\infty} I_{m}^{(1) T E} \mathbf{h}_{m}^{(1) T E}(x, y)=
$$




$$
\sum_{m=1}^{\infty} I_{m}^{(2) T M_{\mathbf{h}_{m}}^{(2) T M}}(x, y)+\sum_{m=1}^{\infty} I_{m}^{(2) T E} \mathbf{h}_{m}^{(2) T E}(x, y)
$$

This last equation can be rearranged adding and subtracting the following terms

$$
\sum_{m=1}^{\infty} \pm V_{m}^{(\delta, \lambda)} Y_{m}^{(\delta, \lambda)} \mathbf{h}_{m}^{(\delta, \lambda)}(x, y)
$$

where $\lambda$ may take the value 1 or 2 for $T E$ and $T M$ modes respectively and $\delta$ again is 1 for region 1 and 2 for region 2 . In the resulting equation the modal currents and voltages can be defined leading to the equivalent network representation shown in fig. 2 and the following final expression for the initial boundary condition.

$$
\sum_{m=1}^{\infty} \sum_{\delta=1}^{2} \sum_{\lambda=1}^{2}(-1)^{\delta-1} \bar{I}_{m}^{(\delta, \lambda)} \mathbf{h}_{m}^{(\delta, \lambda)}(x, y)=\sum_{m=1}^{\infty} \sum_{\delta=1}^{2} \sum_{\lambda=1}^{2} \bar{V}_{m}^{(\delta, \lambda)} Y_{m}^{(\delta, \lambda)} \mathbf{h}_{m}^{(\delta, \lambda)}(x, y)
$$

The formulation of the integral equation is completed by recalling the transform relation between the magnetic field and the modal currents, namely:

$$
\bar{V}_{m}^{(\delta, \lambda)}=\int_{c . s .}\left[\mathbf{z}_{0} \times \mathbf{E}\left(\mathbf{x}^{\prime}, \mathbf{y}^{\prime}\right)\right] \cdot \mathbf{h}_{m}^{(\delta, \lambda) *}\left(x^{\prime}, y^{\prime}\right) \cdot d s^{\prime}
$$

Due to the linearity of the problem we can expand the field in the following form:

$$
\left[\mathbf{z}_{0} \times \mathbf{E}\left(\mathbf{x}^{\prime}, \mathbf{y}^{\prime}\right)\right]=\sum_{p=1}^{\infty} \sum_{\delta=1}^{2} \sum_{\lambda=1}^{2}(-1)^{\delta-1} \bar{I}_{p}^{(\delta, \lambda)} \mathbf{M}_{p}^{(\delta, \lambda)}\left(x^{\prime}, y^{\prime}\right)
$$

On using equations (6), (7) and (8), the solution of the problem can be expressed in mathematical terms with the following integral equation

$$
\begin{aligned}
\mathbf{h}_{p}^{(\eta)}(x, y)=\int_{c . s .} \mathbf{M}_{p}^{(\eta)}\left(x^{\prime}, y^{\prime}\right) \cdot & {\left[j \omega \epsilon_{0} \sum_{m} \sum_{\delta=1}^{2} \hat{Y}_{m}^{T M(\delta)} \mathbf{h}_{m}^{T M(\delta)}(x, y) \mathbf{h}_{m}^{T M(\delta) *}\left(x^{\prime}, y^{\prime}\right)+\right.} \\
& \left.\frac{1}{j \omega \mu_{0}} \sum_{m} \sum_{\delta=1}^{2} \hat{Y}_{m}^{T E(\delta)} \mathbf{h}_{m}^{T E(\delta)}(x, y) \mathbf{h}_{m}^{T E(\delta) *}\left(x^{\prime}, y^{\prime}\right)\right] d s^{\prime}
\end{aligned}
$$

together with the expression for the coupling matrix elements

$$
Z_{m, p}^{(\xi, \eta)}=\int_{c . s .} \mathbf{M}_{p}^{(\eta)}\left(x^{\prime}, y^{\prime}\right) \cdot \mathbf{h}_{m}^{(\xi) *}\left(x^{\prime}, y^{\prime}\right) \cdot d s^{\prime}
$$

where $\eta$ and $\xi$ indicate $T E$ or $T M$ modes and in waveguide 1 or waveguide 2 according to fig. 1 . It is important to note that the only frequency dependent part in the kernel of equation (9) is the factor $\omega$, since $\mathbf{h}(x, y)$ is the vector mode function of the modes and therefore independent from frequency. The integral equation in (9) is solved using a Galerkin algorithm and due to the very simple frequency dependence of the kernel, the final solution for the multimode impedance matrix elements can be written as

$$
Z_{m, p}^{(\xi, \eta)}=j \omega \mu_{0} \sum_{l} \sum_{k=1}^{n} \frac{C_{l, k}^{(\xi, \eta)(m, p)}}{1-\omega^{2} \epsilon_{0} \mu_{0} \omega_{k}}
$$

where the coefficients $C_{l, k}^{(\xi, \eta)(m, p)}$ and $w_{k}$ depend only on the geometry and therefore need to be computed only once. The frequency dependence of the coupling matrix elements is then simply obtained by performing the sum in equation (11) for each point in frequency. This frequency extraction procedure can lead to a considerable time saving for the analysis of complex waveguide structures. In fact, complex devices can be represented in terms of a linear system that combines all the various multimode network representations without any intermediate matrix inversion. The resulting global system needs to be inverted only once for each point in frequency and, since it has a band structure, 
the calculation can be performed very rapidly.

\section{APPLICATIONS}

As a validation of the theory developed, in fig. 3 we present a comparison between the data obtained with our approach and the data presented in [5], including measurements. As it can be observed the agreement with our results is good even for the higher order modes.

The software developed has then been used to design a commercial communication satellite filter structure composed of the cascade of an 8-pole band pass filter, a 6-pole low pass filter, and two double plane step transformers. The complete structure was designed to operate in C-band, being the useful bandwidth: $5.925 \mathrm{GHz}$ to $6.425 \mathrm{GHz}$.

First, the band pass filter and the low pass filter were optimized separately. The design procedure was then completed by optimizing the complete structure using the two double plane transformer as the key element for the connection between the two filters. The hardware was manufactured in two alumina blocks (E-plane cut) usign milling machine technique, and subsequent silver plating of the resulting pieces. The manufacturing tolerances were better than 20 microns in the worst case.

In fig. 4 the in band s-parameters of the complete structure are presented and compared with measurements done on the manufactured hardware. Fig. 5 shows in detail the in band insertion losses while fig. 6 shows the group delay of both simulations and measurements. As it can be seen, better than $0.25 \mathrm{~dB}$ of insertion losses and better than $23 \mathrm{~dB}$ of return losses was achieved in the useful bandwidth. In addition, out of band results are also presented in fig. 7. As it can be seen in all cases the agreement between measurements and the simulated results obtained with our approach is very good.

The strong far out of band requirements for this particular application made necessary the use of the low pass filter, in order to eliminate the spurious responses of the band pass filter alone. In particular, the whole structure was designed to give a rejection better than $30 \mathrm{~dB}$ in the band from $12 \mathrm{GHz}$ to $15 \mathrm{GHz}$. Measured results of the complete hardware in this frequency range are presented in fig. 8 , showing how indeed the rejection of $30 \mathrm{~dB}$ has been succesfully achieved in the whole band.

The analysis of the structure was done on an IBM RISC 6000 platform. A first version of the code has been implemented solving directly equation (9) with the method of moments, and using (10) for the computation of the coupling impedances. It took 1 minute and 39 seconds to characterize all the structure (composed of 36 discontinuities). This characterization needs to be performed only once since it depends only on the geometry and not on frequency (the kernell of (9)). Additionally, for each point in frequency it took 2.5 seconds to get the $s$ parameters of the structure. This time was divided in 2 seconds to invert the method-of-moment system plus computation of the coupling matrix for the 36 discontinuities, plus 0.5 seconds to invert the final network. The subsequent implementation of the frequency*extraction, using equation (11), is currently in progress and, as we can see, it holds potential for substantial further reduction in computation time since it would significantly reduce the 2 seconds now needed to invert the method-of-moment system.

\section{CONCLUSIONS}

In this paper a new method for the analysis of arbitrary two dimensional junctions of rectangular waveguides is presented. The method is based on the extension the Multimode Equivalent Network theory to a two dimensional problem. Comparisons with measured data indicate that the resulting network representation is indeed very accurate. Furthermore, because of the particular formulation of the problem, the resulting code can be computationally very efficient.

\section{References}

[1] M. Guglielmi and G. Gheri. "Rigorous Multimode Network/Numerical Representation of Inductive Step". Accepted for publication on IEEE Transactions on Microwave Theory and Tech- 
niques.

[2] M. Guglielmi and G. Gheri. "Rigorous Multimode Network Representation of Capacitive Steps". Accepted for publication on IEEE Transactions on Microwave Theory and Techniques.

[3] H.K. Smith, J.R. Mosig and M .Guglielmi. "Multimode Network Approach for the Solution of Two Dimensional Waveguide Discontinuities". 23rd European Microwave Conference. Madrid. Spain, 6-9 September 1993.

[4] N. Marcuvitz. "Waveguide Handbook". IEE Electromagnetic waves series 21. Editors: P.J.B. Claricots, E.D.R. Shearman and J.R. Wait.

[5] Hartmut Patzelt, Fritz Arndt. "Double-Plane Steps in Rectangular Waveguides and their Application for Transformer, Irises, and Filters". IEEE Transactions on Microwave Theory and Techniques, Vol MTT-30, No. 5, May 1982.

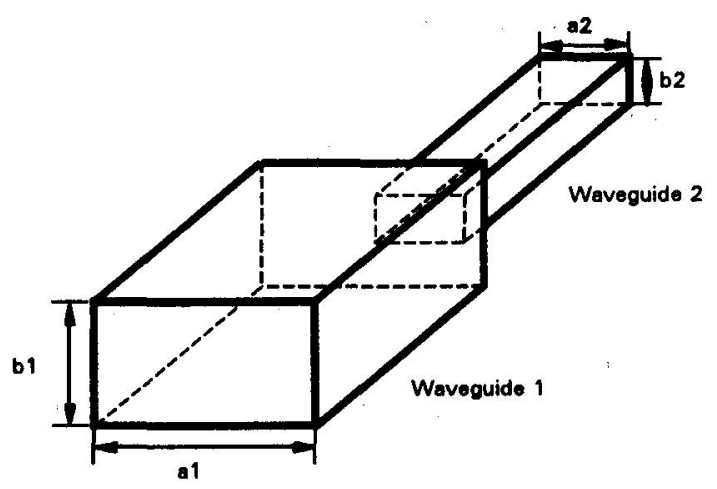

Fig. 1 Two dimensional step in a rectangular waveguide studied in this paper.

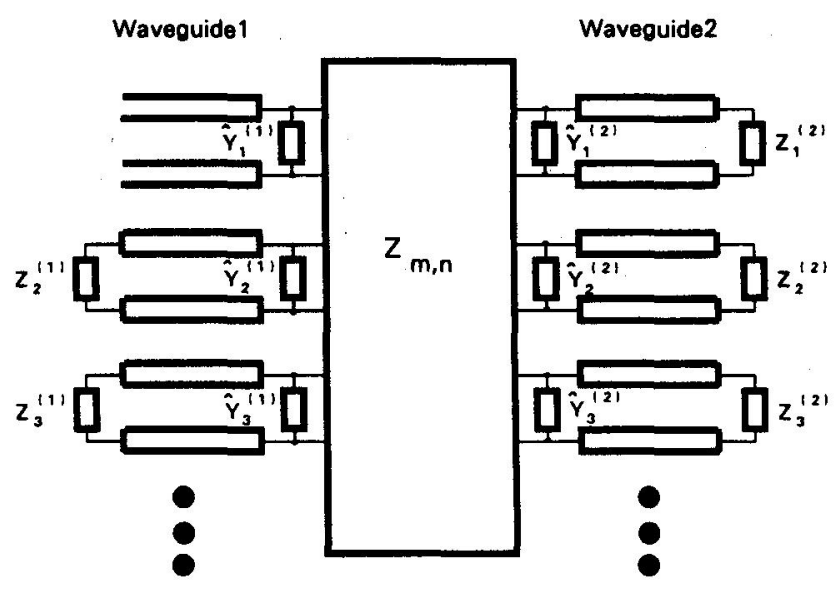

Fig. 2 Multimode Equivalent Network representation of the discontinuity shown in fig. 1 . 


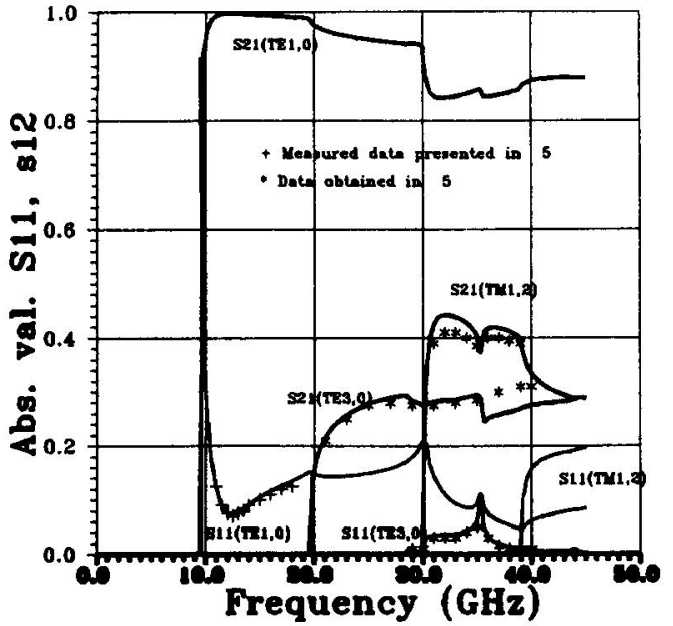

Fig. 3 Comparison between data obtained with our method and data presented in [5]. The structure

is a single centered step with $a_{1}=15.8 \mathrm{~mm}, b_{1}=7.9 \mathrm{~mm}$, $a_{2}=22.9 m m, b_{2}=10.2 m m(a$ and $b$ are shown in fig.1).

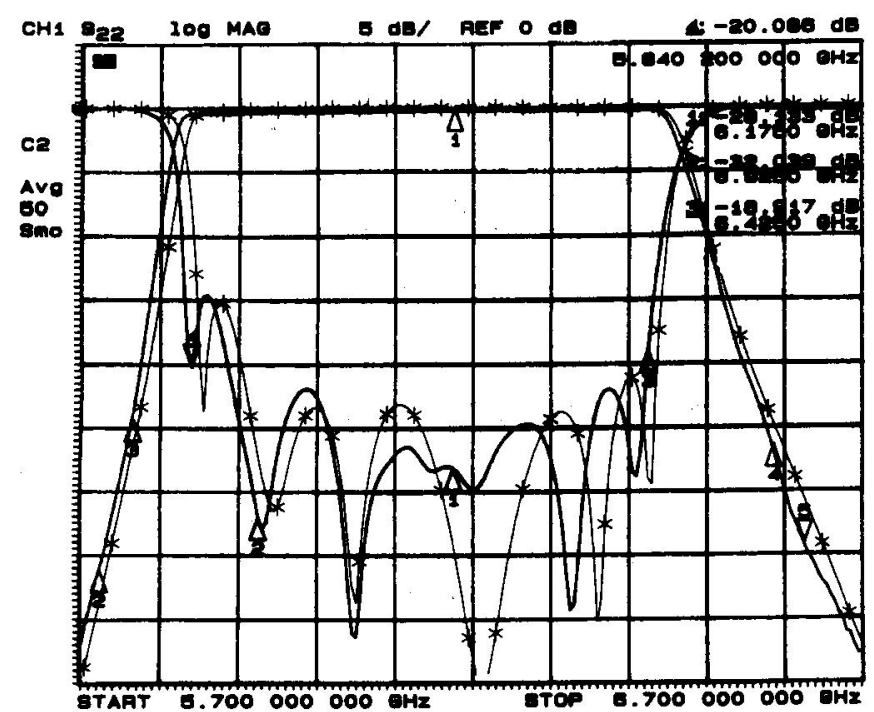

Fig. 4 In band response of the complete structure analyzed in this paper. The structure is composed by an 8-pole band pass filter, 6 -pole low pass filter and two double plane step transformer. The thin line with the stars represents the simulated results while the thick line represents the measurement of the hardware.

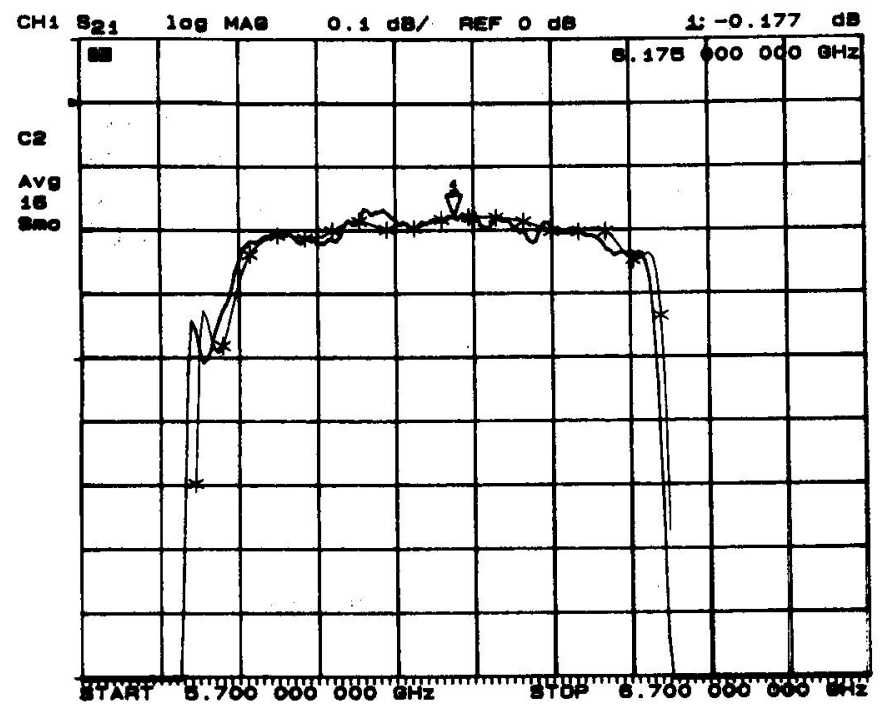

Fig. 5 Detailed insertion losses for the same structure as in fig. 4 . The thin line with the stars represents the simulated results while the thick line represents the measurement of the hardware. 


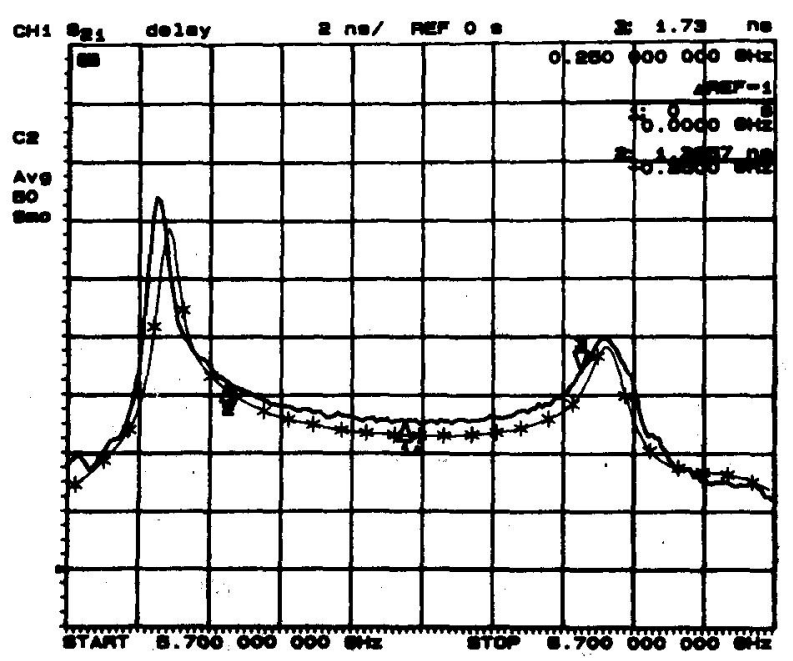

Fig. 6 Group delay of the complete structure as indicated in fig. 4. The thin lin" with the star represents the simulated results while the thick line represents the measurement of the hardware

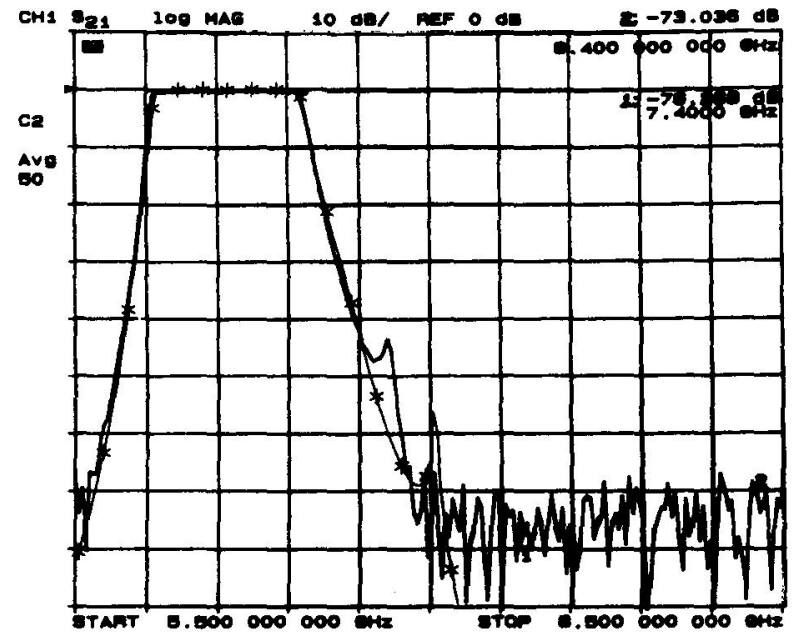

Fig. 7 Response of the complete structure up to 8.5 Gill The thin line with the stars represents the simulated results while the thick line represents the measurement of the hardware.

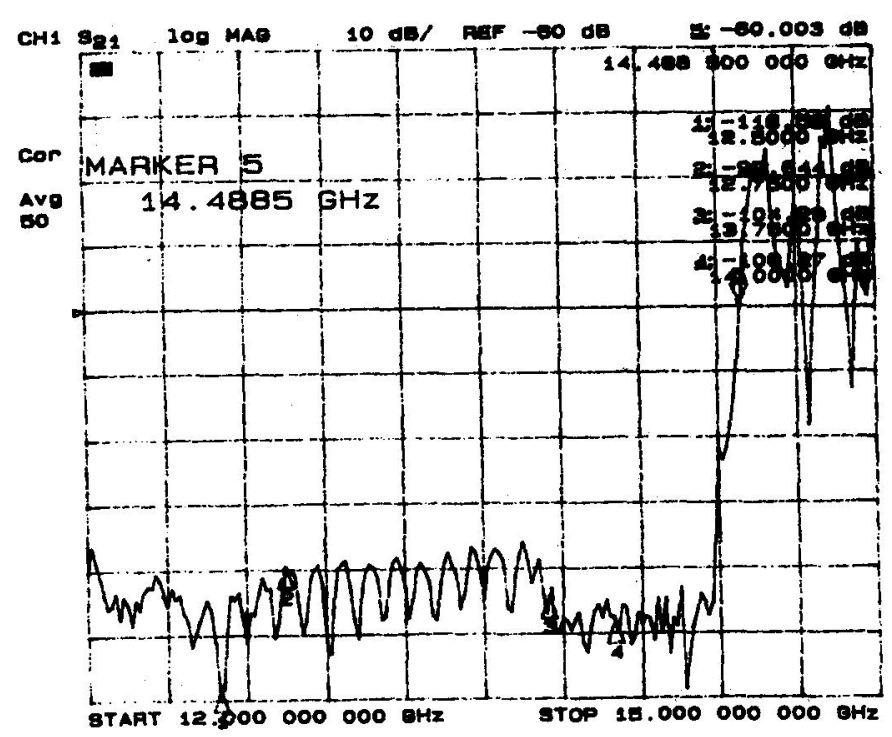

Fig. 8 Response of the complete structure in the band $12 \mathrm{GHz}$ to $15 \mathrm{GHz}$. Due to the effect of the low pass filter the rejection is better than $30 \mathrm{~dB}$ over the whole band. 